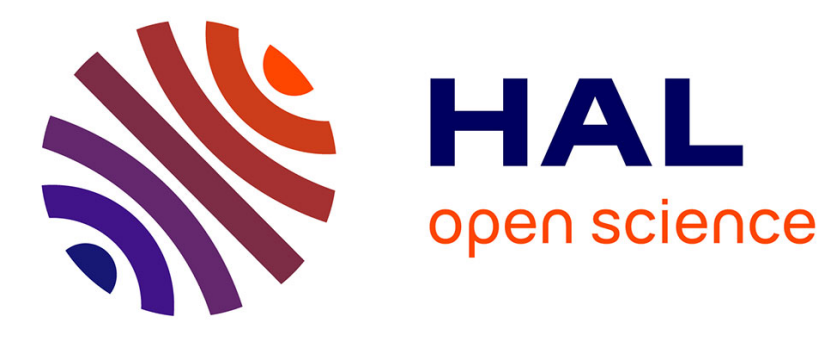

\title{
Structure of liquid ammonia at high pressures and temperatures
}

Jean-Antoine Queyroux, Sandra Ninet, Gunnar Weck, Gastón Garbarino, Mohamed Mezouar, Frédéric Datchi

\section{- To cite this version:}

Jean-Antoine Queyroux, Sandra Ninet, Gunnar Weck, Gastón Garbarino, Mohamed Mezouar, et al.. Structure of liquid ammonia at high pressures and temperatures. Physical Review B: Condensed Matter and Materials Physics (1998-2015), 2019, 100, pp.224104. hal-02382281

\section{HAL Id: hal-02382281 \\ https://hal.science/hal-02382281}

Submitted on 27 Nov 2019

HAL is a multi-disciplinary open access archive for the deposit and dissemination of scientific research documents, whether they are published or not. The documents may come from teaching and research institutions in France or abroad, or from public or private research centers.
L'archive ouverte pluridisciplinaire HAL, est destinée au dépôt et à la diffusion de documents scientifiques de niveau recherche, publiés ou non, émanant des établissements d'enseignement et de recherche français ou étrangers, des laboratoires publics ou privés. 


\title{
Structure of liquid ammonia at high pressures and temperatures
}

\author{
Jean-Antoine Queyroux, ${ }^{1, *}$ Sandra Ninet, ${ }^{1}$ Gunnar Weck, ${ }^{2}$ \\ Gaston Garbarino, ${ }^{3}$ Mohamed Mezouar, ${ }^{3}$ and Frédéric Datchi ${ }^{1, \dagger}$ \\ ${ }^{1}$ Institut de Minéralogie, de Physique des Matériaux et de Cosmochimie (IMPMC), Sorbonne Université, \\ CNRS UMR 7590, IRD UMR 206, MNHN, 4 place Jussieu, F-75005 Paris, France \\ ${ }^{2}$ CEA, DAM, DIF, F-91297 Arpajon, France \\ ${ }^{3}$ European Synchrotron Radiation Facility, Boîte Postale 220, 38043 Grenoble, France
}

(Dated: October 15, 2019)

\begin{abstract}
The structure of liquid ammonia $\left(\mathrm{NH}_{3}\right)$ is investigated from 1 to $6.3 \mathrm{GPa}$ and up to $800 \mathrm{~K}$ by means of synchrotron x-ray diffraction (XRD) and ab initio molecular dynamics (AIMD) simulations. The XRD data are used to extract the molecular structure factor $S_{m o l}(Q)$, pair distribution function $(\mathrm{PDF}) g_{m o l}(r)$, and the density of $\mathrm{NH}_{3}$. There is an excellent agreement between present $S_{m o l}(Q)$ and $g_{m o l}(r)$ at our lowest density and those reported in reference neutron experiments. Our densities agree better with the equation of state of Tillner-Roth et al. [1] than with more recent equation of state (EoS) models. The experimental structure factor and PDF are well reproduced by AIMD simulations using either the BLYP or the PBE exchange-correlation functional. The shapes of $S_{m o l}(Q)$ and $g_{m o l}(r)$ vary little over the investigated pressure range and suggest a compact liquid with weak orientational correlations between molecules, which is corroborated by the coordination number varying from 12.7 to $\sim 14$. The simulations are used to study the evolution of the site-site pair distribution functions, which reveals that the number of $\mathrm{H}$-bonds per molecule (between 1.5 and 2) do not evolve with density, and that the distribution of $\mathrm{H}$ atoms around $\mathrm{N}$ atoms becomes more and more anisotropic with pressure.
\end{abstract}

PACS numbers: Valid PACS appear here

\section{INTRODUCTION}

Ammonia is a major component of the giant icy planets and their moons and has many uses in the chemical industry, which explains the large amount of research devoted to its study. At the fundamental level, a longstanding interest in ammonia arises from its ability to form hydrogen $(\mathrm{H})$ bonds. The $\mathrm{H}$-bond refers to the interaction between a hydrogen atom and a negatively polarized atom (the acceptor $\mathrm{A}$ ), such that a bridge, noted $\mathrm{D}-\mathrm{H} \cdots \mathrm{A}$, is formed between the atom to which the hydrogen is bonded (the donor $\mathrm{D}$ ) and the atom $\mathrm{A}$. It is well known that the properties of water are determined by the $\mathrm{O}-\mathrm{H} \cdots \mathrm{O}$ H-bond. Without it the melting and boiling points of $\mathrm{H}_{2} \mathrm{O}$ would be more than $200 \mathrm{~K}$ lower and life as we know would be impossible. The hydrogen bonds are also responsible for the diversity of polymorphs known for water ice.

The H-bonds in $\mathrm{NH}_{3}$ have been described as weaker and less directional than those formed by $\mathrm{H}_{2} \mathrm{O}$, and their relevance to explain the structural motifs formed by $\mathrm{NH}_{3}$ molecules is disputed. In the gas phase, $\mathrm{NH}_{3}$ has been found to be a good $\mathrm{H}$-bond acceptor but a poor donor [2]. In the solid phase I, stable below $195 \mathrm{~K}$ at ambient pressure, the $\mathrm{N}$ atoms adopt a pseudo face-centered cubic (fcc) arrangement and each molecule has 12 neighbors in the first shell [3]. Among the latter, 6 are at a $\mathrm{N}-\mathrm{N}$ distance of $0.3375 \mathrm{~nm}$ and the 6 others are at 0.3940

\footnotetext{
*queyroux@protonmail.ch

$\dagger$ frederic.datchi@sorbonne-universite.fr
}

$\mathrm{nm}$. Those at the shorter distances have been described as H-bonded to the central molecule, 3 as acceptor and 3 as donor. Contrary to water ice H-bonds, these bonds are non-linear (the bond angle is $160^{\circ}$ ) and weaker than in water ice -a single lone electronic pair is shared by $3 \mathrm{H}$-bonds, and their existence was actually questioned by Ref. [2]. However both high-resolution x-ray diffraction [4] and ab initio molecular dynamics (AIMD) calculations [5] show that the electron density distribution along the bond is consistent with $\mathrm{H}$-bonding. It is more difficult to state on the presence of H-bonds in the plastic solids II and III, which occur at higher pressures along the melting line, due to their large orientational disorder. Above $3.6 \mathrm{GPa}$ at $293 \mathrm{~K}$, ammonia transforms to another proton-ordered phase, phase IV, whose structure was determined by neutron diffraction [6]. In this phase, the $\mathrm{N}$ atoms have a pseudo hexagonal close-packed arrangement, and as in phase I, $6 \mathrm{H}$-bonds may be assigned to each molecule. But in contrast to phase I, the three $\mathrm{N}-\mathrm{D} \cdots \mathrm{H}$ bonds are all different in length and angle. This asymmetry increases at higher pressure, in particular above the transition to the isosymmetric phase $\mathrm{V}$, where the shortest and thus strongest $\mathrm{H}$-bond becomes nearly linear at $178^{\circ}$, while the two others remain bent at $150^{\circ}$ and $160^{\circ}$ [7]. In summary the presence of $\mathrm{H}$ bonds in the ordered solid phases is well established, but unlike $\mathrm{H}_{2} \mathrm{O}$, they do not have a strong impact on the solid structures - except probably for imposing proton ordering, since all of them are close to compact molecular arrangements.

The situation is less clear-cut in the liquid phase. Evidence for hydrogen bonding have been obtained from 


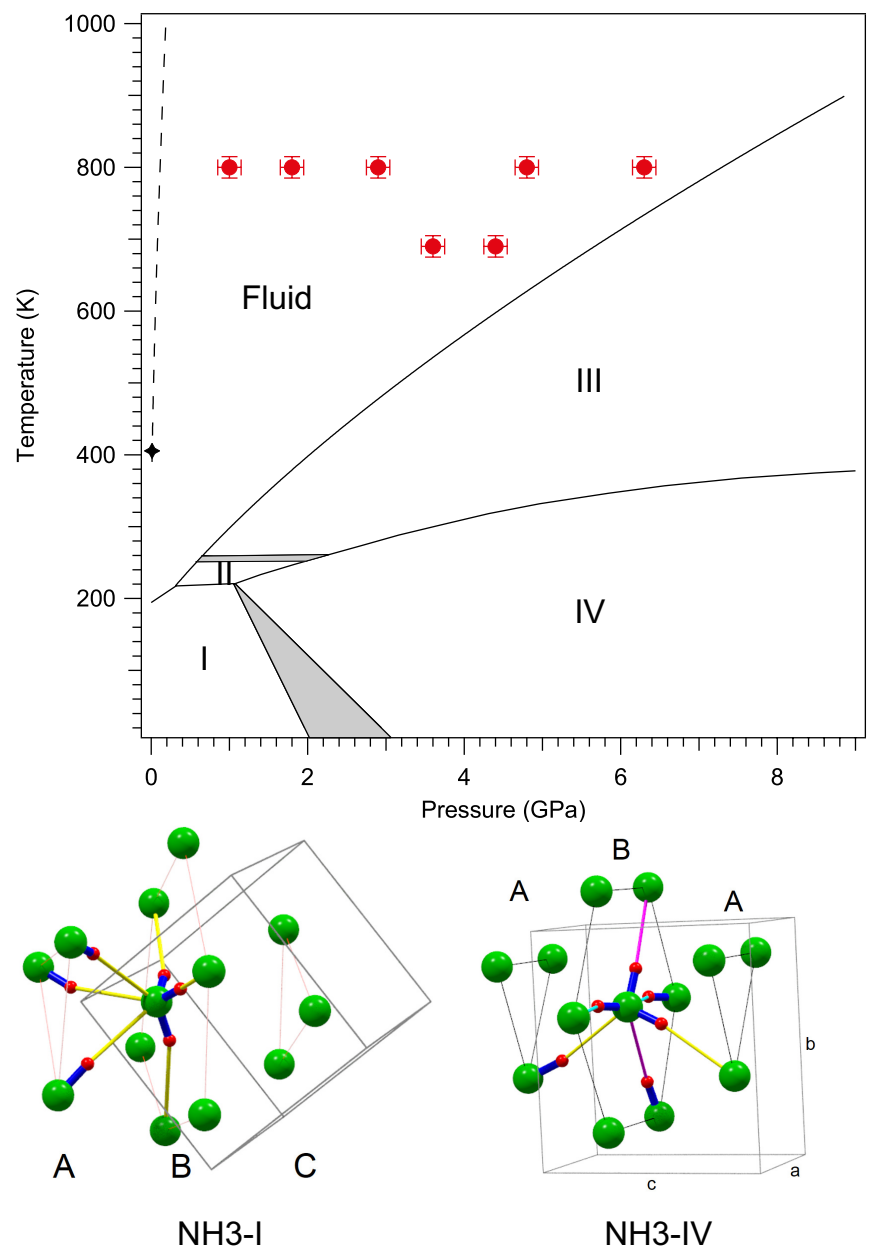

FIG. 1. (Top) Phase diagram of $\mathrm{NH}_{3}$ after Ninet and Datchi [8]. Red circles show the P-T points where experimental data were taken, black star is the critical point and dashed line is the Frenkel line. (Bottom) Representation of the structures of $\mathrm{NH}_{3}-\mathrm{I}$ and $\mathrm{NH}_{3}-\mathrm{IV}$. Colored lines show hydrogen bonds, with different colors for bonds of different lengths and angles.

neutron diffraction [9-12], elastic [13, 14] and inelastic $\mathrm{x}$-ray scattering [15] experiments, as well as from theoretical simulations [16-20]. The N-N pair distribution function at $277 \mathrm{~K}$ obtained by the x-ray diffraction experiment of Ref. [14] presents a maximum at $0.34 \mathrm{~nm}$ and a shoulder at $0.37 \mathrm{~nm}$, which, by analogy with the solid phase I, may be interpreted as the respective signature of $\mathrm{H}$-bonded and non-bonded contacts, and thus suggests a well-structured first shell. More recent neutron diffraction experiments and simulations found, however, that the liquid is much less structured. They indicate that an ammonia molecule forms on average $\sim 2 \mathrm{H}$-bonds with its neighbors. These H-bonded molecules appear on the N-H(D) pair distribution function (PDF) as a shoulder at $\sim 0.25 \mathrm{~nm}$ of the strong oscillation peaked at $\sim 0.4$ $\mathrm{nm}$. Nevertheless, the spatial distribution of the nitrogen atoms shows that no extended H-bonded network exists in the ammonia liquid since the first solvation shell con- tains 12 to 13 molecules. Again, this is in sharp contrast with the case of liquid water where the solvation structure of a molecule is determined primarily by hydrogen bonding interactions.

Except for the recent work of Guthrie et al. [12], all previous experimental studies of the structure of liquid ammonia have been carried out at or near ambient pressure. For water, several neutron [21, 22] and x-ray diffraction experiments $[23,24]$ have been reported to pressures of several GPa. They showed that the density increase between ambient and $1 \mathrm{GPa}$ has a large effect on the structure, disrupting the 3D network of H-bonds although preserving the tetrahedral $\mathrm{H}$-bonding scheme around a given molecule. In ammonia, the comparison between the structure at ambient and $1 \mathrm{GPa}$ did not show a strong difference in the short-range structure, however a substantial increase of the spatial correlations at large distance was found [12].

The purpose of the present study is to extend the experimental investigation to higher pressures in order to determine the structural evolution over a larger range of densities. To this end, we used recently developed techniques for the measurement of the structure of low- $\mathrm{Z} \mathrm{flu-}$ ids in the diamond anvil cell by synchrotron x-ray diffraction [25], which have been successfully used in the recent past to study liquid hydrogen [26] and carbon dioxide [27] and allowed to determine the melting lines of liquid nitrogen [28] and ammonia [29] up to the megabar range. Here we present experimental data for liquid ammonia obtained at $690 \mathrm{~K}$ and $800 \mathrm{~K}$ up to $6.3 \mathrm{GPa}$. The data is analyzed to extract both the x-ray molecular structure factor and pair distribution function. Experimental data are compared to $a b$ initio calculations performed in the same pressure-temperature (P-T) range as experiments.

\section{EXPERIMENTAL METHODS}

\section{A. Experiments}

Ammonia samples (99.99\%, Air Liquide) were loaded cryogenically into a membrane diamond anvil cell (DAC) equipped with diamond anvils of Boehler-Almax design [30], $300 \mu \mathrm{m}$ culet size and $70^{\circ} 4 \theta$ x-ray aperture. A rhenium foil of $200 \mu \mathrm{m}$ thickness was used as gasket and pre-indented to $80 \mu \mathrm{m}$. To prevent any chemical reaction, the ammonia sample was separated from rhenium by a gold liner of thickness about $5 \mu \mathrm{m}$. The latter was also used as the in situ pressure sensor, using the P-V-T equation of state (EoS) of gold from Ref. [31]. The pressures obtained with other EoS [32-37] deviate by less than 0.5 $\mathrm{GPa}$ in this pressure range. The DAC was heated with a ring-shaped external resistive heater enclosing the DAC and temperatures were determined with a type-K thermocouple (made of Chromel and Alumel alloys) in contact with the diamond anvil. The estimated pressure and temperature uncertainties are $0.15 \mathrm{GPa}$ and $15 \mathrm{~K}$, respectively. 
Angular dispersive x-ray diffraction experiments were carried out at the ID27 beamline of the European Synchrotron Radiation Facility (ESRF, Grenoble, France). The x-ray beam of wavelength $0.3738 \AA(33 \mathrm{keV})$ was focussed to produce a spot size of $3 \times 3 \mu \mathrm{m}$ on the sample. A MAR345 image plate was used for detection. Diffraction patterns were integrated using the Dioptas software [38].

The main difficulty of present experiments is to extract the very weak coherent signal scattered by the sample from the incoherent (Compton) scattering originating from the $1.7 \mathrm{~mm}$-thick diamond anvils. In order to reduce this background, a multichannel collimator (MCC) was used as described in Ref. [25]. The MCC is composed of two concentric sets of circular slits positioned between the sample and the image plate whose effect is to isolate a diffracting volume centered on the sample. The slits are carefully aligned to make their rotation center coincide with the sample. In order to cover all diffraction angles the MCC is rotated over the angular distance separating two adjacent slits during the collection. To further limit the parasitic noise coming from the experimental hutch, the MCC is enclosed by a lead cover extending up to the detector. The spatial selectivity of the slits increases with the diffraction angle and reduces the anvil contribution by a factor $\sim 100$ at $20 \mathrm{~nm}^{-1}$ and $\sim 400$ at $80 \mathrm{~nm}^{-1}$. The disadvantage of the MCC is the loss of signal due to the absorption by the slits and to their limited height which only covers $16^{\circ}$ in the vertical direction. The acquisition time is thus increased relative to experiments without the MCC and was set here at $300 \mathrm{~s}$. The background (empty cell) signal was measured at $300 \mathrm{~K}$ after pressure was released and the sample escaped from the gasket hole.

\section{B. Data analysis}

In order to process the measured intensity $I^{\text {meas }}(Q)$ ( $Q=4 \pi \sin \theta / \lambda$ is the momentum transfer) and obtain a molecular structure factor $S_{\text {mol }}(Q)$ and a molecular pair distribution function $g_{m o l}(r)$, we use the methodology presented in details in Eggert et al. [39] and Weck et al. [25], which we briefly recall below.

The measured signal can be written as follows:

$$
I^{\text {meas }}(Q)=T^{D A C}(Q) T_{\text {samp }}^{M C C}(Q) I^{\text {samp }}(Q)+s I^{b k g}(Q)
$$

where $T^{D A C}(Q)$ is the DAC transmission, $T_{\text {samp }}^{M C C}(Q)$ is the MCC transmission for the sample scattering. They are calculated analytically using the known geometry of the DAC and MCC, as explained in Weck et al. [25]. $I^{\text {samp }}(Q)$ is the total scattering from the sample, $I^{b k g}(Q)$ is the background signal originating mainly from the Compton scattering of the diamond windows, and $s$ is a scaling factor representing the ratio of the incident $\mathrm{x}$ ray beam intensity used for the measurements of $I^{b k g}(Q)$ and $I^{\operatorname{samp}}(Q)$. Since it is not possible to measure $s$ with high enough accuracy, it is taken as a fit parameter in the analysis [39]. The background contribution was obtained by measuring the scattered signal from the empty cell at the end of the experiment. $I^{\text {samp }}(Q)$, can be expressed as the sum of the coherent and incoherent scattering, $I^{c o h}(Q)$ and $I^{\text {incoh }}(Q)$, respectively,

$$
I^{s a m p}(Q)=\frac{1}{N \alpha}\left[I^{c o h}(Q)+N \sum_{p} I_{p}^{i n c o h}(Q)\right]
$$

where $\alpha$ is the normalization factor used to express the sample scattering in atomic units and $I_{p}^{\text {incoh }}(\mathrm{Q})$ is the incoherent Compton scattering from atoms of type $p$ in the sample computed using the analytic atomic formulas given by Hajdu [40]. The molecular structure factor is defined as:

$$
S_{m o l} \equiv \frac{I^{c o h}(Q)}{N Z_{t o t}^{2} f_{e}^{2}(Q)}
$$

Where $f_{e}(Q)$ is an effective electronic form factor defined as $f_{e}(Q)=\left(f_{N}(Q)+3 f_{H}(Q)\right) / Z_{t o t}$, where $f_{N}(Q)$ is the atomic form factor for $\mathrm{N}$ computed using the analytic atomic formulas given by Hajdu [40] while the atomic form factor for $\mathrm{H}$ is directly calculated from exact theoretical expressions [41]. $S_{\infty}=\left(K_{N}^{2}+3 K_{H}^{2}\right) / Z_{\text {tot }}^{2}$, where $K_{X}(X=N, H)$, is an average effective atomic number defined as the average over the integration $Q$-range of the functions $K_{X}(Q)=f_{X}(Q) / f_{e}(Q)$.

The molecular pair distribution function $g_{m o l}(r)$ is then obtained by the inverse Fourier transform

$$
\begin{aligned}
F(r) & \equiv 4 \pi \rho\left[g_{\text {mol }}(r)-1\right] \\
& =\frac{2}{\pi} \int_{0}^{\infty} Q i(Q) \sin (Q r) d Q
\end{aligned}
$$

where $i(Q)=S_{m o l}(Q)-S_{\infty}$ and $\rho$ is the average molecular density.

The normalization coefficient $\alpha$ is calculated according the Krogh-Moe [42] and Norman [43] method:

$$
\alpha=Z_{\text {tot }}^{2} \frac{-2 \pi^{2} \rho+\int_{0}^{Q_{\max }}\left[J(Q)+S_{\infty}\right] Q^{2} \mathrm{~d} Q}{\int_{0}^{Q_{\max }}\left[I^{\text {samp }}(Q) / f_{e}^{2}(Q)\right] Q^{2} \mathrm{~d} Q}
$$

where $J(Q)=\sum_{p} I_{p}^{\text {incoh }}(Q) / Z_{\text {tot }}^{2} f_{e}^{2}(Q)$.

Initially, density $\rho$ is not known and Eq. (6) is only exact when $Q_{\max }=\infty$. This makes the determination of $\alpha$ somewhat uncertain. We also need to know the scale factor $s$ with high precision. To determine $\alpha$ and $s$ we therefore use a self-consistent procedure based on a method developed by Kaplow et al. [44] and refined in Ref. [39]. The idea is to remove the nonphysical oscillations in $F(r)$, dominant at low $r$, coming from errors in 
$\alpha$ and $s$. To this end, we use the fact that for $r$ values below the minimum intermolecular distance (noted $r_{\min }$ ), $F(r)$ should match the theoretical form:

$$
F^{\text {theo }}(r)=-4 \pi \rho+F^{\text {intra }}(r) \text { for } r<r_{\min }
$$

where $F^{\text {intra }}(r)$ corresponds to the intramolecular contribution to $F(r)$. A frozen molecule formulation is chosen for $F^{\text {intra }}(r)$, which considers the molecule as a rigid body with a fixed $(\mathrm{N}, \mathrm{H})$ distance of $1.1024 \AA$ and $(\mathrm{H}, \mathrm{H})$ distance of $1.6242 \AA$ [45]. This approximation should be good enough in the pressure range of present experiments. The difference $\Delta F(r)$ between the initial $F(r)$ from Eq. (4) and $F^{\text {theo }}(r)$ is calculated and Fourier transformed to evaluate the correction to $i(Q)$. The new $F(r)$ is then calculated using Eq. (4), and the procedure is iterated until convergence. As noted by Kaplow et al. [44] and Eggert et al. [39], $\rho$ is an independent variable in this analysis with a direct influence on $\alpha$ and $\Delta F(r)$ so that $\rho$ can be extracted by minimizing $\Delta F(r)$. The optimum value for the scale factor $s$ can similarly be extracted. Thus $\rho$ and $s$ are varied to minimize $\Delta F(r)$ using a $\chi^{2}$ figure of merit defined as $\chi^{2}=\int_{0}^{r_{\min }}[\Delta F(r)]^{2} d r$. During this optimization, a damping function of the form $\exp \left(-A Q^{2}\right)$ is used in the Fourier transform to reduce the termination effects from the finite value of $Q_{\max }$, but the final $S_{m o l}(Q)$ and $g_{m o l}(r)$ are calculated without the use of damping.

We also note that in order to determine the transmission of the sample scattering through the MCC, the knowledge of the sample thickness is required. This cannot be measured at present so it is taken as an additional fit parameter in the procedure [25].

Finally, in order to compare our $S_{\text {mol }}(Q)$ and $g_{m o l}(r)$ with other experimental and theoretical data, we use the following relations:

$$
\begin{aligned}
S_{m o l}(Q)= & \left(K_{N}^{2} S_{N N}(Q)+9 K_{H}^{2} S_{H H}(Q)+\right. \\
& \left.6 K_{N} K_{H} S_{N H}(Q)\right) / Z_{t o t}^{2}+S_{\infty}
\end{aligned}
$$

$g_{\text {mol }}(r)=\frac{K_{N}^{2} g_{N N}(r)+9 K_{H}^{2} g_{H H}(r)+6 K_{N} K_{H} g_{N H}(r)}{Z_{\text {tot }}^{2}}$

where $S_{\alpha \beta}$ and $g_{\alpha \beta}$ are the partial structure factors and partial pair distribution functions (PDF), respectively. For $Q_{\max }=90 \mathrm{~nm}^{-1}, K_{N}=8.4085$ and $K_{H}=0.5305$, thus the respective contributions of $g_{N N}(r), g_{N H}(r)$ and $g_{H H}(r)$ to $g_{m o l}(r)$ are $70.7 \%, 26.8 \%$ and $2.5 \%$.

\section{Ab initio molecular dynamics}

We conducted Born-Oppenheimer molecular dynamics simulations at 8 different densities using the PW code of

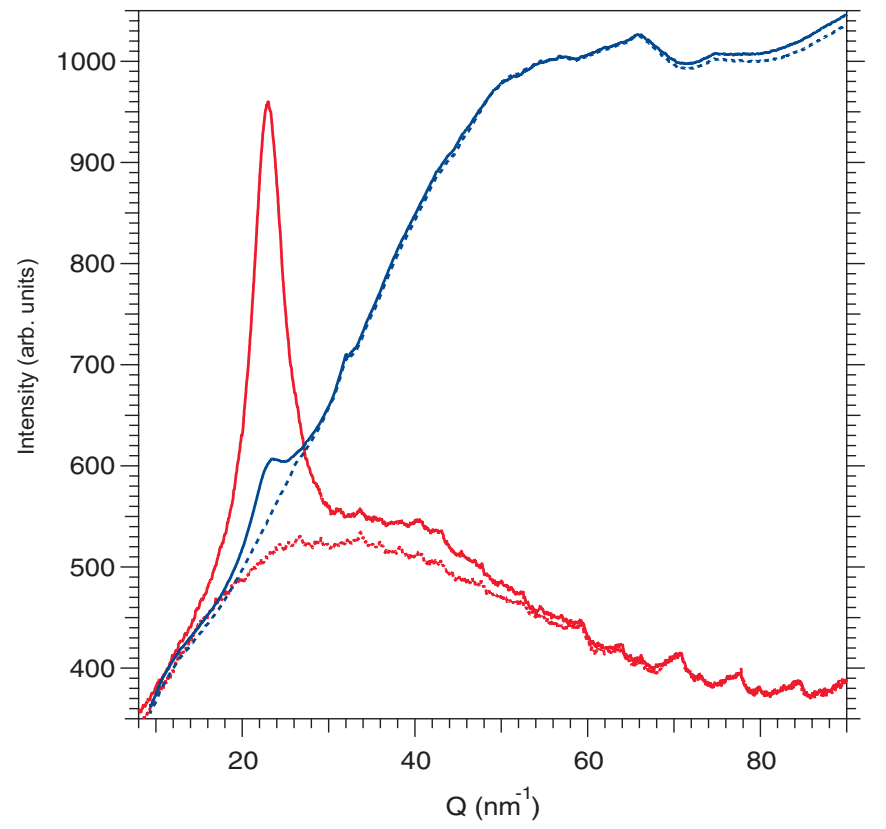

FIG. 2. Integrated x-ray diffraction patterns from a $\mathrm{NH}_{3}$ fluid sample at $4.3 \mathrm{GPa}, 690 \mathrm{~K}$, with and without the MCC. The red and blue solid lines are patterns obtained respectively with and without the MCC. The red and blue dashed lines are the respective patterns from the empty cell with and without MCC. The intensities for the patterns obtained without MCC have been divided by 20 to scale with those measured with the MCC.

the Quantum ESPRESSO package [46]. Most simulations used the Perdew-Burke-Ernzerhof (PBE) [47] exchange correlation functional with a plane wave energy cutoff of $800 \mathrm{eV}$, since PBE functional was found to provide very good agreement with experiment for the equation of state [48] and Raman modes [49] of solid ammonia at high pressure. We also compared the PBE functional results to those obtained with the Becke-Lee-Yang-Parr (BLYP) functional $[50,51]$ at three different densities (29, 35 and 39.4 molecules.nm ${ }^{-3}$ ), as BLYP functional was found to agree well with the structural data of the ambient pressure liquid $[17,18]$. Brillouin zone was sampled at the $\Gamma$ point only. The temperature was equilibrated with a Berendsen thermostat [52].

A supercell containing $108 \mathrm{NH}_{3}$ molecules was used. The system was equilibrated at two different temperatures (690 and $800 \mathrm{~K}$ ) and each pressure was run in the NVT ensemble with a Berendsen thermostat during 10 ps.

\section{RESULTS \& DISCUSSION}

\section{A. Experiments}

In total, seven x-ray diffraction patterns of fluid $\mathrm{NH}_{3}$ were collected from 1.0 to $6.3 \mathrm{GPa}$ at $690 \mathrm{~K}$ and $800 \mathrm{~K}$. 


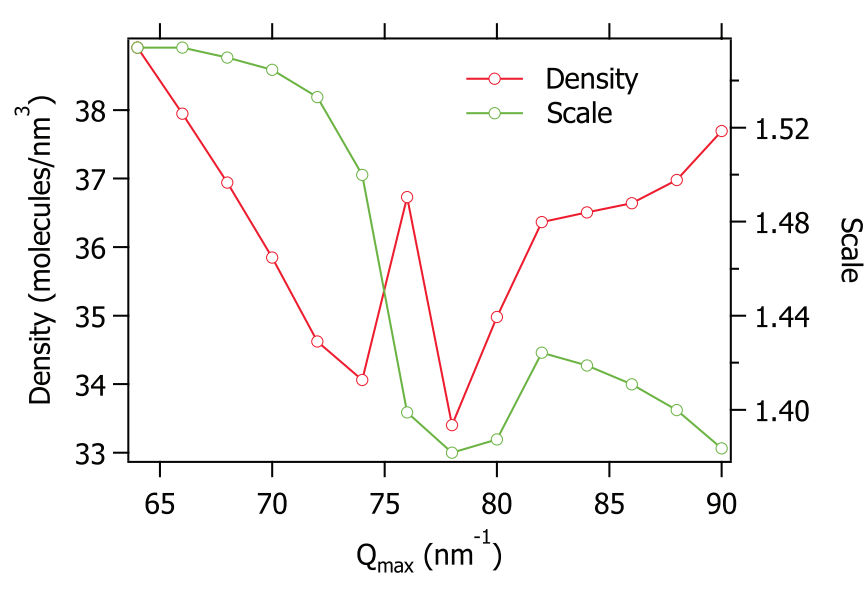

FIG. 3. Optimum density and scale factor for ammonia at 4.8 $\mathrm{GPa}$ and $800 \mathrm{~K}$ as a function of $Q_{\max }$. The average density is $36.3 \pm 1.5$ molecules. $\mathrm{nm}^{-3}$.

At these $P-T$ condition, ammonia is in the supercritical state (the critical point of $\mathrm{NH}_{3}$ is at $11.3 \mathrm{MPa}, 405.5 \mathrm{~K}$ ). To determine whether the data set crosses the Frenkel line delimiting the nonrigid (or gas-like) from the rigid (or liquid-like) states [53], we estimated the latter using the sound-velocity minimum criterion and the temperature dependence of the sound velocity from the Nationnal Institute of Standards and Technology database [45], as in Trachenko et al. [54]. As can be seen in Fig. 1, the presure along the Frenkel line remains below $0.11 \mathrm{GPa}$ for $T<800 \mathrm{~K}$, thus all our data points correspond to liquid-like states.

Fig. 2 illustrates the raw signal obtained from a sample of fluid $\mathrm{NH}_{3}$ at $4.3 \mathrm{GPa}, 690 \mathrm{~K}$ obtained with and without the MCC. It can be observed that without the MCC, the signal of liquid ammonia cannot be distinguished from that of the empty cell above $30 \mathrm{~nm}^{-1}$, while with the MCC, the signal stands above the background up to $\sim 70$ $\mathrm{nm}^{-1}$.

Nonetheless, the use of the MCC introduces parasitic oscillations due to mechanical imperfections of the MCC, which become more and more visible with increasing $Q$ as the liquid signal becomes very weak. These oscillations are only partly removed when subtracting the empty cell contribution, thus in order to reduce their effects on the data treatment, such as artificial ripples in the pair distribution function, the sample scattering intensity is smoothed using a cubic spline smoothing routine which allows the amount of smoothing to vary as a function of $\mathrm{Q}[55]$.

The procedure outlined above to process the raw data requires to fix some parameters $\left(Q_{\max }, r_{\min }\right.$, smoothing and damping factors), while the others are obtained from the optimization process (scale factor $s$ and normalization factor $\alpha$, sample thickness $s_{t h}$ ).

One of the most critical parameter is the maximum $Q$ value, $Q_{\max }$, to which the data is used. Although experimental data were collected up to $100 \mathrm{~nm}^{-1}$, the rapid decrease of signal-to-noise ratio with $Q$ imposed to

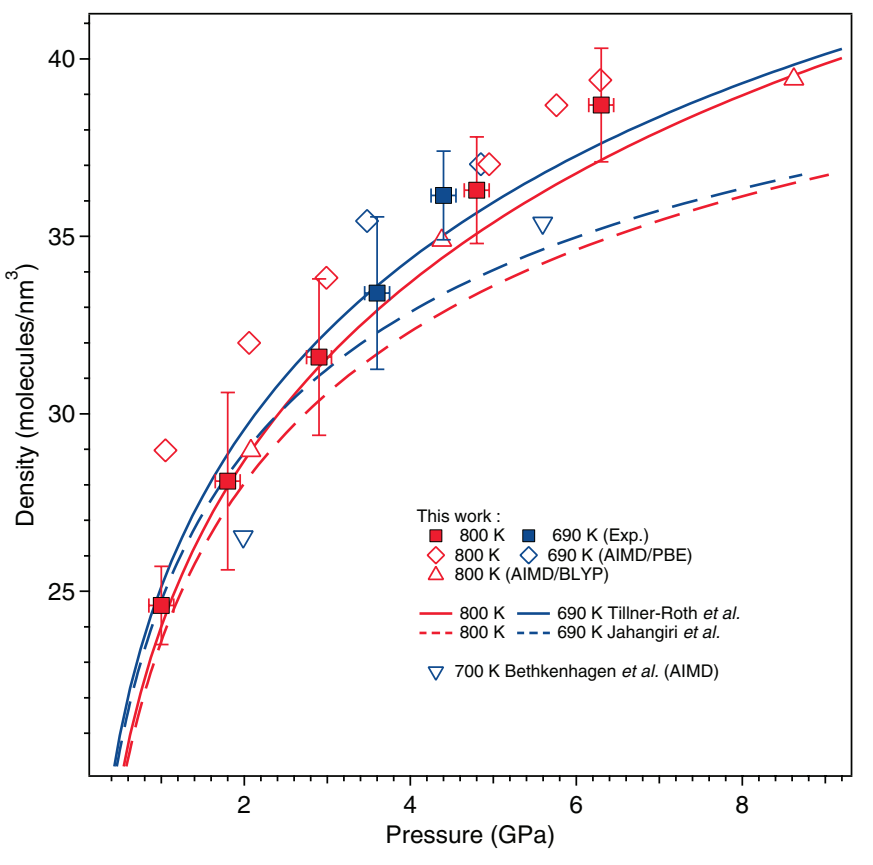

FIG. 4. Evolution with pressure of the density of liquid ammonia. Red and blue colours are for $T=800 \mathrm{~K}$ and $T=690 \mathrm{~K}$, respectively. Squares are experimental data, lozenges are from AIMD simulations with the PBE functional, and up-triangles are from AIMD simulations with the BLYP functional. The solid and dashed lines are the densities obtained with the EoS of Tillner-Roth et al. [1] and Jahangiri and Behnejad [56], respectively. Blue down triangles are taken from the AIMD simulations of Bethkenhagen et al. [57] at $700 \mathrm{~K}$.

set $Q_{\max }$ to a lower value. We found here that the best compromise was obtained by setting $Q_{\max }$ at $90 \mathrm{~nm}^{-1}$, except for the lowest density data $(1 \mathrm{GPa}, 800 \mathrm{~K})$ where $Q_{\max }$ was set at $65 \mathrm{~nm}^{-1}$.

The optimum values of $\rho$ and $s$ obtained by the minimization of the low- $r$ oscillations in $F(r)$ are also sensitive to $Q_{\max }$. For our final $\rho$, we report the average values obtained by varying $Q_{\max }$ in a range where $S_{\text {mol }}(Q)$ and $g_{m o l}(r)$ are well behaved, and use the standard deviations as error bars [39]. The variation of the number density and scale factor when $Q_{\max }$ is varied between 64 and 90 $\mathrm{nm}^{-1}$ is shown in Fig. 3 for the pattern at $4.8 \mathrm{GPa}$ and $800 \mathrm{~K}$. The average value at this pressure is $36.3 \pm 1.5$ molecules.nm ${ }^{-3}$.

The number density obtained for the complete data set is plotted against pressure in Fig. 4. In the range of present experiment, the density continuously increases by $57 \%$ from $24.6 \pm 1.1$ to $38.7 \pm 1.6$ molecules.nm ${ }^{-3}$. To our knowledge, there is no other reported experimental data in the literature in this $P-T$ range. We thus compare our experiments to semi-empirical EoS, on one hand, and to the results of our AIMD simulations, on the other hand. The first semi-empirical EoS which we examined is that of Tillner-Roth et al. [1], since this EoS is nowadays considered as the reference $\mathrm{EoS}$ for liquid $\mathrm{NH}_{3}$. 

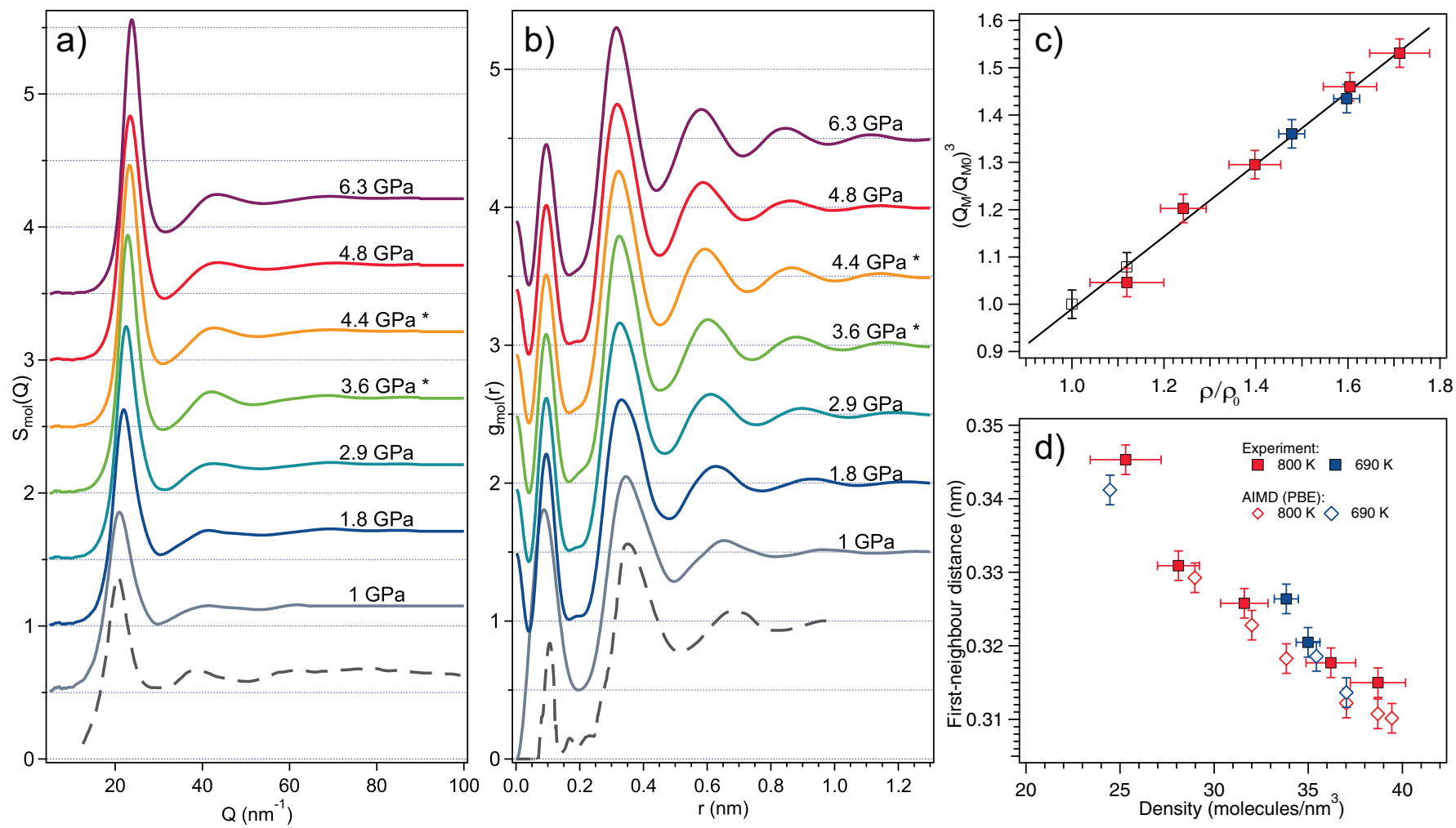

FIG. 5. Experimental results from the present x-ray diffraction study of liquid $\mathrm{NH}_{3}$. Panels (a) and (b) show respectively the molecular structure factor and pair distribution functions obtained at the indicated pressures. The curves are offset by 0.5 for clarity. The temperature was $800 \mathrm{~K}$ except for the patterns labelled by the ${ }^{*}$ symbol, for which temperature was $690 \mathrm{~K}$. The dashed lines are $S_{m o l}(Q)$ and $g_{m o l}(r)$ calculated using the experimental data from Ricci et al. [11] at $273 \mathrm{~K}$ and $0.483 \mathrm{MPa}$. the Panel (c) shows $\left(Q_{M} / Q_{M 0}\right)^{3}$ as a function of $\rho / \rho_{0}$, where $Q_{M}$ is the position of the main diffraction peak, $\rho$ is the liquid density, $Q_{M 0}=20.65 \mathrm{~nm}^{-1}$ and $\rho_{0}=22.6$ molecules.nm ${ }^{-3}$ correspond to the values of liquid ammonia at $273 \mathrm{~K}$ and $0.483 \mathrm{MPa}$ reported by Ricci et al. [11]. Filled and open squares are experimental data from the present work and Ref. [11], respectively. Blue and red are for $T=690 \mathrm{~K}$ and $T=800 \mathrm{~K}$, respectively. The solid line is a linear fit to the data. Panel (d) shows the first-neighbor distance as a function of density, determined as described in the text. Squares are present experimental data, and lozenges are from AIMD simulations with the PBE functional.

It is based on the parametrization of the free energy using a large set of experimental data for the thermodynamic properties of liquid ammonia up to $1 \mathrm{GPa}$ and $720 \mathrm{~K}$. The second one is from the recent work of Jahangiri and Behnejad [56] and is based on a model developed by Song and Mason [58] using the statistical-mechanical perturbation theory of hard spheres. The latter EoS was found to fairly agree with the AIMD results of Bethkenhagen et al. [57] up to $20 \mathrm{GPa}, 3000 \mathrm{~K}$, and predicts a less compressible liquid than Tillner-Roth et al. [1]'s EoS, the difference between the two reaching 3 molecules.nm ${ }^{-3}$ (8 $\%)$ at $7 \mathrm{GPa}$. Our experimental densities are in very good agreement with the EoS of Tillner-Roth et al. [1] up to $\sim 4 \mathrm{GPa}$, then we find higher densities by up to 1 molecules.nm ${ }^{-3}$ at $6.3 \mathrm{GPa}$, but this difference remains within the experimental uncertainties. By contrast, the densities given by the EoS of Jahangiri and Behnejad [56] are too low and the difference with our data exceeds the error bars. This is consistent with the fact that the AIMD densities of Bethkenhagen et al. [57] also underestimates the experimental values. By contrast, our AIMD simulations using the PBE functional predict too high densities at low pressures but the agreement with experiment improves with pressure. This is consistent with the good agreement observed between DFT calculations of the EoS of solid $\mathrm{NH}_{3}$ using the PBE functional [48]. We finally note that for a given density, the AIMD simulations with the BLYP functional give higher pressures than those using the PBE functional, and the resulting $P-\rho$ data closely follows the EoS of Tillner-Roth et al. [1] in the covered $P-T$ range.

The evolution of $S_{m o l}(Q)$ with pressure is shown in Fig. $5(\mathrm{a})$. At all pressures, the molecular structure factor of fluid $\mathrm{NH}_{3}$ is dominated by a first diffraction peak (FDP) located at $21 \mathrm{~nm}^{-1}$ at $1 \mathrm{GPa}, 800 \mathrm{~K}$, followed by broad oscillations. The main effects of increasing pressure is to sharpen the diffraction peaks, increase their intensity and shift them to higher $\mathrm{Q}$. The maximum value of the FDP varies from 1.36 to $2.06(+51 \%)$ and shifts in Q by $13.3 \%$ from 1 to $6.3 \mathrm{GPa}$. Fig. 5(a) also shows the $S_{\text {mol }}(Q)$ of liquid ammonia at $273 \mathrm{~K}$ close to the saturation pressure $\left(\rho=22.6\right.$ molecules.nm $\left.{ }^{-3}\right)$, which was calculated using 


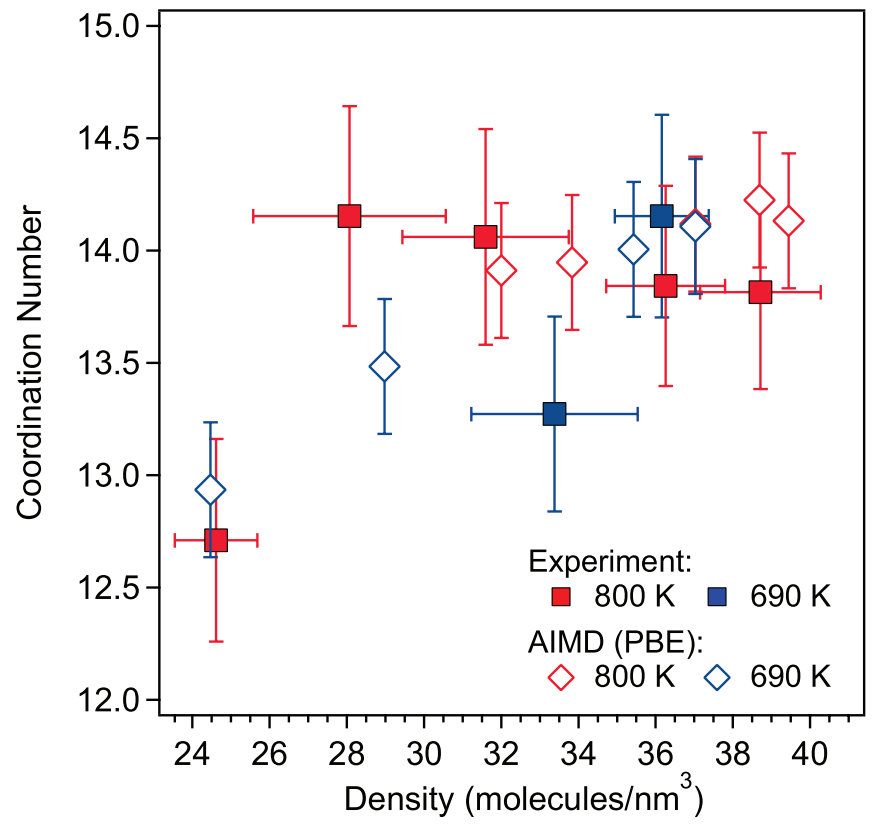

FIG. 6. Evolution with density of the coordination number in liquid $\mathrm{NH}_{3}$. Squares and lozenges represent experimental and AIMD results, respectively. Red and blue are for $T=800 \mathrm{~K}$ and $690 \mathrm{~K}$, respectively.

Eq. 8 and the partial structure factors reported by Ricci et al. [11]. There is a good correspondance between this $S_{\text {mol }}(Q)$ and the one we determined at $1 \mathrm{GPa}, 800 \mathrm{~K}$ $\left(\rho=24.6 \pm 1.1\right.$ molecules.nm $\left.{ }^{-3}\right)$, except that the latter has broader peaks, which is likely due to the effect of the higher temperature. The shape of $S_{m o l}(Q)$ is at first glance, very similar to that of a liquid with no or weak orientational correlations such as $\mathrm{H}_{2}[26,59], \mathrm{N}_{2}$ [60, 61] and $\mathrm{O}_{2}[61,62]$. In dense monatomic liquids it is empirically found that the position of the maximum of the FDP, $Q_{M}$ is related to the density $\rho$ through the relation $Q_{M} \simeq 4.4(4 \pi \rho / 3)^{1 / 3}[63]$, and thus $\left(Q_{M} / Q_{M 0}\right)^{3} \simeq \rho / \rho_{0}$ where $Q_{M 0}$ and $\rho_{0}$ correspond to a reference state. This relation has also been found to be verified in molecular liquids with weak orientational correlations, such as $\mathrm{H}_{2}[26]$ and $\mathrm{N}_{2}[28]$. To test whether it also holds in ammonia, we plot in Fig. 5(c), $\left(Q_{M} / Q_{M 0}\right)^{3}$ as a function of $\rho / \rho_{0}$, using the data of Ricci et al. [11] at $273 \mathrm{~K}, 0.483$ $\mathrm{MPa}$ as the reference state. It can be seen that these two quantities are linearly related in liquid ammonia, but the slope of $0.76(1)$ is lower than 1 , thus indicating that liquid $\mathrm{NH}_{3}$ somewhat differs from a simple liquid. It can also be noticed that the $Q_{M}^{3}$ data fall on the same line, irrespective of the temperature, and are thus primarily dependent on density.

Figure $5 \mathrm{~b}$ shows the evolution of $g_{m o l}(r)$ with pressure. The first peak in $g_{m o l}(r)$ at $\sim 0.1 \mathrm{~nm}$ corresponds to the intramolecualr N-H peak and is fixed by our data analysis. Its broadening is entirely due to the truncation of the Fourier transform at $Q_{\max }$ and can thus serve as a measure of the experimental resolution. The $\mathrm{H}$ -
$\mathrm{H}$ intramolecular peak at $0.16 \mathrm{~nm}$ cannot be observed, most likely because it is too weak. Similarly as $S_{m o l}(Q)$, the shape of $g_{m o l}(r)$ is not much affected by pressure in the range of present experiments, the main effects being again a sharpening and intensification of the oscillations as they move to lower $r$. Consistently, we find a good correspondence between the $g_{m o l}(r)$ of Ricci et al. [11], calculated using Eq. 9, with our lowest density data. A noticeable difference can however be observed in the low$r$ part which is more structured in Ricci et al. [11]'s data, which we believe is due to the lower resolution of our data (see also the comparison with AIMD simulations below). The present x-ray data also confirm the absence of the oscillations at $0.37 \mathrm{~nm}$ and $0.46 \mathrm{~nm}$ reported by Narten [14] which were not observed in the neutron diffraction studies [9-12].

The position of the first intermolecular peak approximately gives the distance between first neighbours, and is plotted versus density in Fig. 5(d). This distance varies from 0.345 to $0.315 \mathrm{~nm}$ over the experimental pressure range. The ratio between the positions of the first and the second peak are 2 and 1.8 for the lowest and the highest pressure reached in this experiment, respectively. These values characterize a liquid without extended preferential orientation, which is consistent with the observations made above.

Figure 6 shows the evolution of the average coordination number of ammonia molecules with density. This quantity was determined by integrating $4 \pi r^{2} \rho g_{m o l}(r)$ up to the first minimum of $g_{m o l}(r)$. Using our AIMD simulations, we checked that the difference in coordination number obtained by using $g_{m o l}(r)$ instead of $g_{N N}(r)$ is below $+6 \%$. It is seen that the coordination number slowly increases with density, going from 12.7 to 14.1. These numbers are similar to those found earlier in the neutron diffraction studies (14 in Ricci et al. [11]) and indicate a close packing of molecules at all pressures.

Our experimental data thus suggest that the structure of liquid $\mathrm{NH}_{3}$ is weakly affected by pressure. Unfortunately, the interpretation of the data in terms of structure is limited because $g_{m o l}(r)$ is a weighted sum of three partial PDFs (Eq. 9) which cannot be extracted from a single diffraction pattern. In the following, we compare our experimental data to AIMD simulations and use the latter to obtain greater details on the liquid structure.

We first start by comparing the AIMD predictions for the partial PDFs using the PBE functional, on one hand, and the BLYP functional, on the other hand. This is illustrated in Fig. 7(a) for the highest density of our data set, $\rho=39.4$ molecules.nm ${ }^{-3}$, and for $T=800 \mathrm{~K}$. We find an almost exact correspondence between the two sets of partial PDFs. These two functionals thus give identical results in terms of liquid structure at a given density, however the predicted pressure is higher $(8.6 \mathrm{GPa})$ for BLYP than for PBE functional (6.3 GPa). Next, we compare the experimental $g_{m o l}(r)$ with the simulated ones, calculated with the help of Eq. 9. This is shown in Fig. 7(b) for four simulation runs at densities close to exper- 

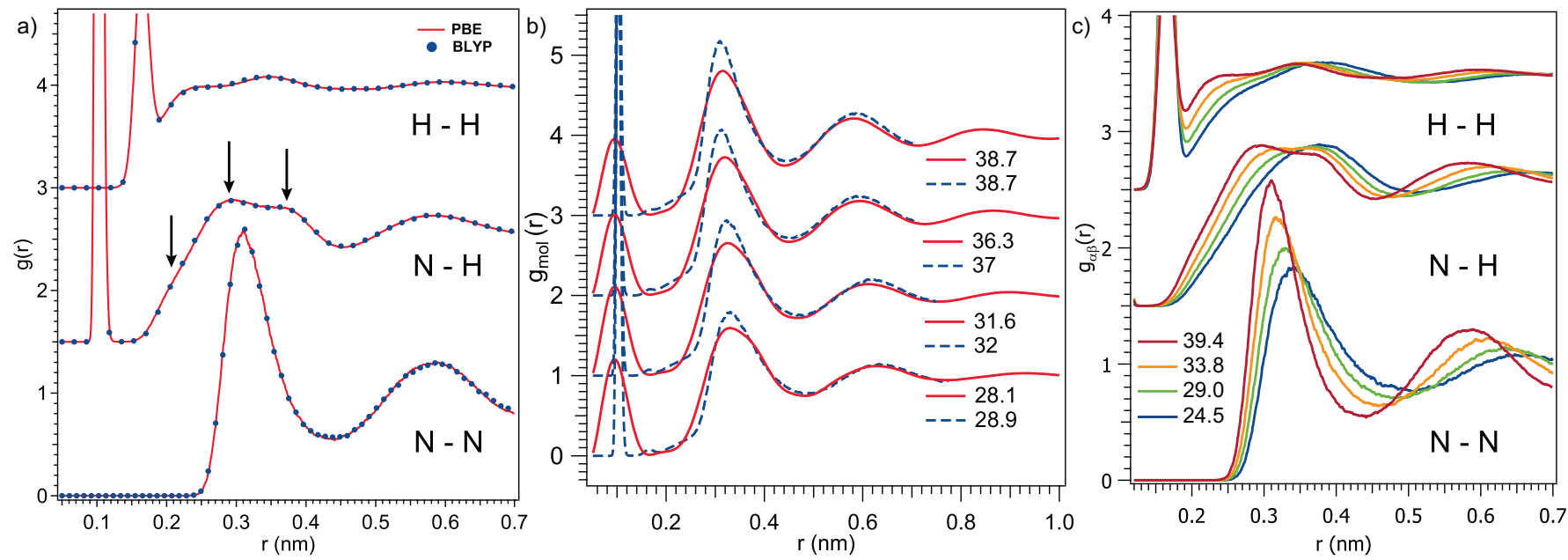

FIG. 7. Results from AIMD simulations. (a) Comparison between the NN, NH and HH partial PDFs obtained using either the PBE or BLYP functional at the fixed density of 39.4 molecules.nm ${ }^{-3}$ and $800 \mathrm{~K}$. Offsets of 1.5 and 3 are applied to NH and $\mathrm{HH}$ distributions respectively. The arrows designate the three humps at $r \simeq 0.21,0.29$ and $0.37 \mathrm{~nm}$ in the NH partial PDF. (b) Comaparison between the experimental (red solid lines) and theoretical (blue dashed lines) $g_{m o l}(r)$ at close densities. The $\rho$ values in molecules $/ \mathrm{nm}^{3}$ are indicated for each curve. The theoretical $g_{m o l}(r)$ were obtained with the PBE functional. Curves are offset by 1. (c) Evolution with density of the theoretical partial PDFs. The blue dotted, green dashed, orange dot-dashed and red solid lines correspond to $\rho$ values of $24.5,29,33.8$ and 39.4 molecules.nm ${ }^{-3}$, respectively. Offsets of 1.5 and 2.5 are applied for $\mathrm{NH}$ and $\mathrm{HH}$ distributions, respectively.

imental ones. There is a fair agreement between the experimental and theoretical $g_{m o l}(r)$ over the probed range of densities: the peak positions are well reproduced, as well as the amplitude of the second oscillation. The first peak is smaller and broader in the experiment, which we think is due, at least in part, to the low $Q_{\max }$ truncation. Indeed the same effects were previously observed in the x-ray study of water $[23,39]$. A noticeable feature is that the non-zero intensity between $\sim 0.18$ and $\sim 0.22 \mathrm{~nm}$, which, as seen later on, is assigned to $\mathrm{NH}$ and $\mathrm{HH}$ contributions, is observed in both experimental and theoretical $g_{m o l}(r)$. Due to the limited size of the simulation box, the theoretical $g_{m o l}(r)$ only extends to 0.7 $\mathrm{nm}$, which was found insufficient to determine meaningful $S_{m o l}(Q)$ by inverse Fourier transform. This prevents a direct comparison with the experimental $S_{m o l}(Q)$ which do not suffer from the " $Q_{\max }$ problem". The good agreement between simulations and experiments also extends to the density evolution of the first-neighbor distance and coordination number, as seen in Fig. 5(d) and Fig. 6.

The present AIMD simulations enable to access the evolution of the partial (or site-site) PDF with density which are shown in Fig. $7(\mathrm{c})$. Like $g_{m o l}(r)$, to which it contributes by $\sim 70 \%, g_{N N}(r)$ presents a first peak which is unstructured and has a similar shape as encountered in simple compact liquids. There is, however, a noticeable asymmetry on the high- $r$ side at low density, which tends to decrease as the peaks sharpen and move to lower $r$ with pressure. More interesting is the evolution of the $\mathrm{NH}$ distribution. At low density, the latter displays a first intermolecular peak with little structure, but on increasing density, several humps appear and the peak seems to be composed of at least three different contributions at $r \simeq 0.21,0.29$ and $0.37 \mathrm{~nm}$. Turning to $g_{H H}(r)$, the first intermolecular peak presents a bimodal distribution at all densities, and the effect of increasing pressure, apart from the general shift, is to increase the intensity of the low- $r$ contribution. These results suggest that, despite the compact nature of the first-neighbour solvation shell, the $\mathrm{H}$ distribution around nitrogen atoms are not isotropic and that orientational correlations exist.

We recall that the presence of $\mathrm{H}$-bonded molecules in the low-pressure, low-temperature liquid was inferred by the presence of a pronounced shoulder in the neutron $g_{N H}(r)$ at $r \simeq 0.235 \mathrm{~nm}$ [11]. In Fig. 8, we compare the experimental partial PDFs of $\mathrm{NH}_{3}$ at $273 \mathrm{~K}, 0.483 \mathrm{MPa}$ determined by Ricci et al. [11] to the AIMD simulations of Boese et al. [18] at the same thermodynamic conditions, on one hand, and to our own simulations at the lowest density $\left(24.5\right.$ molecules.nm $\left.{ }^{-3}\right)$ at $700 \mathrm{~K}$, on the other hand. The simulations of Boese et al. [18] used the BLYP functional and reproduced fairly well the experimental PDFs, except for slight difference in amplitudes. In particular, the shoulder at $\sim 0.235 \mathrm{~nm}$ is present in the NH distribution. Our simulations used the PBE functional, but as seen above, we found no difference between the PDFs produced with the PBE and BLYP functionals. It can be seen that at the higher temperature of our simulations, the shoulder is absent, but the total width of the first intermolecular peak is very similar. This indicates that $\mathrm{H}$ atoms which form $\mathrm{H}$-bonds to the central molecules cannot be distinguished from those which do not, which implies that non-bonded molecules can move closer to the central one in the high temperature liquid. 


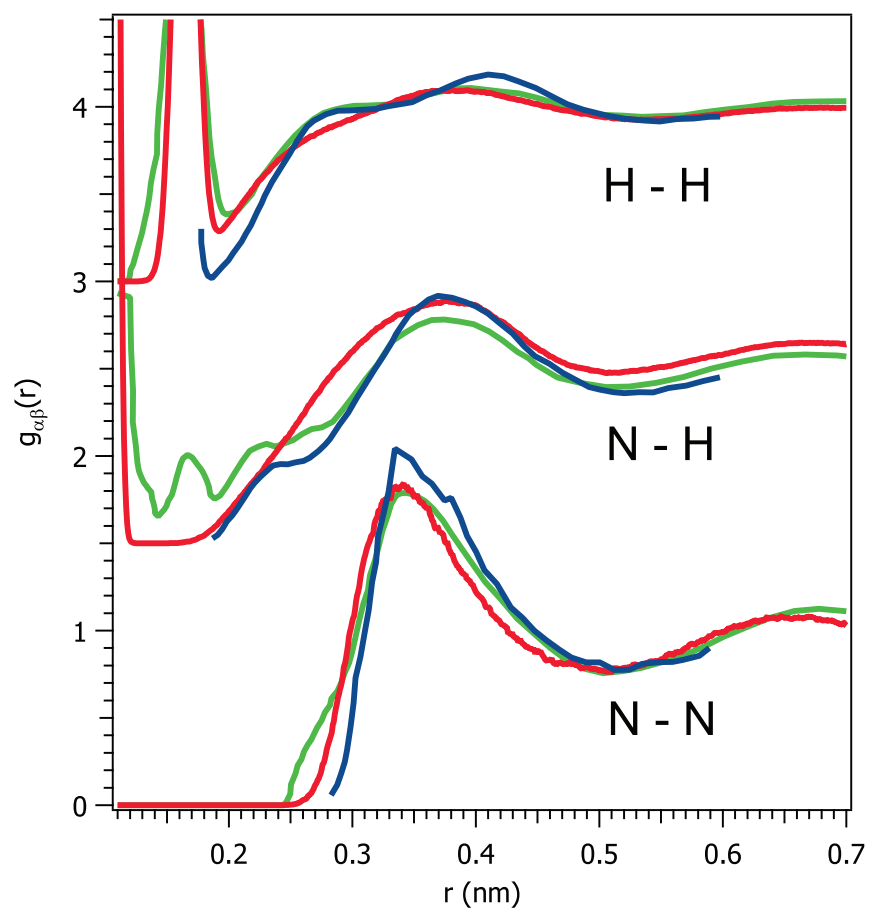

FIG. 8. Partial PDFs of liquid ammonia at low density. The green solid, blue dotted and red dashed lines are, respectively, the experimental data of Ricci et al. [11] at $273 \mathrm{~K}, 0.483 \mathrm{MPa}$, the AIMD simulations of Boese et al. [18] with the BLYP functional at the same $P, T$ conditions, and our AIMD simulations at 24.5 molecules.nm ${ }^{-3}, 700 \mathrm{~K}$ using the PBE functional.

The integration of $4 \pi \rho r^{2} g_{N H}(r)$ using the same boundaries as Ricci et al. [11], i.e from 0.19 to $0.26 \mathrm{~nm}$, gives an average number of $\mathrm{H}$ bonds of $\sim 1.5$ per molecule, instead of $\sim 2$ at $273 \mathrm{~K}$. We note however that the apparent difference with the low-temperature liquid should be taken cautiously as the number of $\mathrm{H}$-bonds are very sensitive to the integration boundaries which are arbitrarily set since there is no resolved H-bond peak.

The shape of the NH distribution found at the highest density (39.4 molecules. $\mathrm{nm}^{-3}$ ) is sensibly different from the ones at low density, as well as from Ricci et al. [11]'s $g_{N H}(r)$ at $273 \mathrm{~K}$. The low- $r$ shoulder assigned to $\mathrm{H}$-bonded $\mathrm{H}$ atoms is recovered, but remains less marked than in the liquid at $273 \mathrm{~K}$. The integration of $4 \pi \rho r^{2} g_{N H}(r)$ up to the inflection point at $\sim 0.235 \mathrm{~nm}$ gives an average of $\sim 2 \mathrm{H}$ bonds per molecule at this density, which is the same as for the low- $T$ liquid, showing that the number of $\mathrm{H}$-bonds per molecule is not much sensitive to density.

\section{CONCLUSION}

In this paper, we have reported the first, to our knowledge, structural data on liquid ammonia above $1 \mathrm{GPa}$ and up to $6.3 \mathrm{GPa}$ at $800 \mathrm{~K}$, using synchrotron x-ray diffraction in a diamond anvil cell. In this $P-T$ range, liquid ammonia is found stable and experience a density increase of $57 \%$. The present experiments made use of advanced filtering techniques enabling to reject a large part of the background scattering from the diamond anvils. This was found crucial in order to extract highquality data for the x-ray molecular structure factor up to $90 \mathrm{~nm}^{-1}$, from which the x-ray molecular pair distribution function was obtained. The present study also enabled to estimate the density of liquid $\mathrm{NH}_{3}$ in a range where no experimental data existed.

The shapes of $S_{m o l}(Q)$ and $g_{m o l}(r)$ were found to little vary with density in the experimental range, and are found similar to that of liquids with weak orientational correlations between molecules. The coordination number varies from 12.7 to $\sim 14$, which is typical of compact liquids. In order to obtain more details of the structure, AIMD simulations using either the PBE or BLYP functional were performed. These two functionals are found to give identical results in terms of pair distribution functions at a given density, but BLYP functional gives higher pressure values than PBE functional. A good agreement is observed between the simulated and experimental $g_{m o l}(r)$, first-neighbor distance and coordination number at the same density. The most significant effect of the density increase is seen on the NH distribution, which displays a first intermolecular peak with no apparent structure at low density while three separate humps appear at high density. The signature of H-bonds is more difficult to observe than for the liquid at low temperature, but the integration of the $\mathrm{NH}$ distribution over the range of expected $\mathrm{H}$-bond distances gives on average from 1.5 to $2 \mathrm{H}$ bonds, similar to the low $T$ liquid.

The present data should be useful to benchmark theoretical models of ammonia in the $10 \mathrm{GPa}, 1000 \mathrm{~K}$ range. Experimentally, the extension of the present work to higher pressures would be very interesting as theoretical studies [57,64] indicate major transformations in the liquid phase above $\sim 20 \mathrm{GPa}$, where molecules start to dissociate. Although x-ray diffraction only allows accessing the total pair distribution functions, such a radical change in the liquid should be easily observed.

\section{ACKNOWLEDGMENTS}

We acknowledge the ESRF for provision of beam time under Long-Term Project HD-463 and the Agence Nationale de la Recherche for financial support under grant ANR 13-BS04-0015 (MOFLEX). 
[1] R. Tillner-Roth, F. Harms-Watzenberg, and H. D. Baehr, DKV-Tagungsbericht, Vol. 20 (1993) p. 167.

[2] D. Nelson Jr., G. Fraser, and W. Klemperer, Science 238, 1670 (1987).

[3] J. W. Reed and P. M. Harris, J. Chem. Phys. 35, 1730 (1961).

[4] R. Boese, N. Niederprüm, D. Bläser, A. Maulitz, M. Y. Antipin, and P. R. Mallinson, J. Phys. Chem. B 101, 5794 (1997).

[5] A. D. Fortes, J. P. Brodholt, I. G. Wood, and L. Vočadlo, J. Chem. Phys. 118, 5987 (2003).

[6] J. S. Loveday, R. J. Nelmes, W. G. Marshall, J. M. Besson, S. Klotz, and G. Hamel, Phys. Rev. Lett. 76, 74 (1996).

[7] S. Ninet, F. Datchi, S. Klotz, G. Hamel, J. S. Loveday, and R. J. Nelmes, Phys. Rev. B 79, 100101 (2009).

[8] S. Ninet and F. Datchi, High pressure-high temperature phase diagram of ammonia., The Journal of chemical physics 128, 154508 (2008).

[9] P. Chieux and H. Bertagnolli, J. Phys. Chem. 88, 3726 (1984).

[10] T. Bausenwein, H. Bertagnolli, A. David, K. Goller, H. Zweier, K. Tödheide, and P. Chieux, J. Chem. Phys. 101, 672 (1994).

[11] M. A. Ricci, M. Nardone, F. P. Ricci, C. Andreani, and A. K. Soper, J. Chem. Phys. 102, 7650 (1995).

[12] M. Guthrie, C. a. Tulk, J. Molaison, and a. M. dos Santos, Phys. Rev. B 85, 184205 (2012).

[13] R. F. Kruh and J. I. Petz, J. Chem. Phys. 41, 890 (1964).

[14] A. H. Narten, J. Chem. Phys. 66, 3117 (1977).

[15] P. Giura, R. Angelini, F. Datchi, G. Ruocco, and F. Sette, J. Chem. Phys. 127, 084508 (2007).

[16] W. L. Jorgensen and M. Ibrahim, J. Am. Chem. Soc. 102, 3309 (1980).

[17] M. Diraison, G. J. Martyna, and M. E. Tuckerman, J. Chem. Phys. 111, 1096 (1999).

[18] A. D. Boese, A. Chandra, J. M. L. Martin, and D. Marx, J. Chem. Phys. 119, 5965 (2003).

[19] A. Tongraar, T. Kerdcharoen, and S. Hannongbua, J. Phys. Chem. A 110, 4924 (2006).

[20] E. A. Orabi and G. Lamoureux, J. Chem. Theory Comput. 9, 2035 (2013).

[21] A. K. Soper, Chem. Phys. 258, 121 (2000).

[22] T. Strässle, A. M. Saitta, Y. Godec, G. Hamel, S. Klotz, J. S. Loveday, and R. J. Nelmes, Phys. Rev. Lett. 96, 67801 (2006).

[23] G. Weck, J. H. Eggert, P. Loubeyre, N. Desbiens, E. Bourasseau, J.-B. Maillet, M. Mezouar, and M. Hanfland, Phys. Rev. B 80, 180202 (2009).

[24] Y. Katayama, T. Hattori, H. Saitoh, T. Ikeda, K. Aoki, H. Fukui, and K. Funakoshi, Phys. Rev. B 81, 14109 (2010).

[25] G. Weck, G. Garbarino, S. Ninet, D. K. Spaulding, F. Datchi, P. Loubeyre, and M. Mezouar, Rev. Sci. Instrum. 84, 063901 (2013).

[26] G. Weck, G. Garbarino, P. Loubeyre, F. Datchi, T. Plisson, and M. Mezouar, Phys. Rev. B 91, 180204 (2015).

[27] F. Datchi, G. Weck, A. M. Saitta, Z. Raza, G. Garbarino, S. Ninet, D. K. Spaulding, J.-A. Queyroux, and M. Mezouar, Phys. Rev. B 94, 014201 (2016).
[28] G. Weck, F. Datchi, G. Garbarino, S. Ninet, J. A. Queyroux, T. Plisson, M. Mezouar, and P. Loubeyre, Phys. Rev. Lett. 119, 235701 (2017).

[29] J. A. Queyroux, S. Ninet, G. Weck, G. Garbarino, T. Plisson, M. Mezouar, and F. Datchi, Phys. Rev. B 99, 134107 (2019).

[30] R. Boehler and K. De Hantsetters, High Pressure Res. 24, 391 (2004).

[31] O. L. Anderson, D. G. Isaak, and S. Yamamoto, J. Appl. Phys. 65, 1534 (1989).

[32] J. C. Jamieson, J. N. Fritz, and M. H. Manghnani, in High-pressure research in Geophysics, edited by S. Akimoto and M. H. Manghnani (Springer Netherlands, 1982) pp. $27-48$.

[33] S. H. Shim, T. S. Duffy, and K. Takemura, Earth Planet. Sc. Lett. 203, 729 (2002).

[34] T. Tsuchiya, J. Geophys. Res-Sol. Ea. 108, 1 (2003).

[35] Y. Fei, A. Ricolleau, M. Frank, K. Mibe, G. Shen, and V. B. Prakapenka, P. Natl. Acad. Sci. USA 104, 9182 (2007).

[36] P. I. Dorogokupets and A. Dewaele, High Pressure Res. 27, 431 (2007).

[37] M. Yokoo, N. Kawai, K. G. Nakamura, K. I. Kondo, Y. Tange, and T. Tsuchiya, Phys. Rev. B 80, 104114 (2009).

[38] C. Prescher and V. B. Prakapenka, High Pressure Res. 7959, 223 (2015).

[39] J. H. Eggert, G. Weck, P. Loubeyre, and M. Mezouar, Phys. Rev. B 65, 174105 (2002).

[40] F. Hajdu, Acta Crystallogr. A 28, 250 (1972).

[41] R. F. Stewart, E. R. Davidson, and W. T. Simpson, J. Chem. Phys. 42, 3175 (1965).

[42] J. Krogh-Moe, Acta Crystallogr. 9, 951 (1956).

[43] N. Norman, Acta Crystallogr. 10, 370 (1957).

[44] R. Kaplow, S. L. Strong, and B. L. Averbach, Physical Review 138, A1336 (1965).

[45] P. J. Linstrom and W. G. Mallard, eds., NIST Chemistry webBook; NIST standard reference database No. 69 (National Institute of Standards and Technology, Gaithersburg MD, 2001).

[46] P. Giannozzi, S. Baroni, N. Bonini, M. Calandra, R. Car, C. Cavazzoni, D. Ceresoli, G. L. Chiarotti, M. Cococcioni, I. Dabo, A. Dal Corso, S. de Gironcoli, S. Fabris, G. Fratesi, R. Gebauer, U. Gerstmann, C. Gougoussis, A. Kokalj, M. Lazzeri, L. Martin-Samos, N. Marzari, F. Mauri, R. Mazzarello, S. Paolini, A. Pasquarello, L. Paulatto, C. Sbraccia, S. Scandolo, G. Sclauzero, A. P. Seitsonen, A. Smogunov, P. Umari, and R. M. Wentzcovitch, J. Phys-Condens. Matter 21, 395502 (2009).

[47] J. P. Perdew, K. Burke, and M. Ernzerhof, Phys. Rev. Lett. 77, 3865 (1996).

[48] F. Datchi, S. Ninet, M. Gauthier, A. Saitta, B. Canny, and F. Decremps, Phys. Rev. B 73, 174111 (2006).

[49] S. Ninet, F. Datchi, A. Saitta, M. Lazzeri, and B. Canny, Phys. Rev. B 74, 104101 (2006).

[50] A. D. Becke, Phys. Rev. A 38, 3098 (1988).

[51] C. Lee, W. Yang, and R. G. Parr, Phys. Rev. B 37, 785 (1988).

[52] H. J. C. Berendsen, J. P. M. Postma, W. F. van Gunsteren, A. DiNola, and J. Haak, J. Chem. Phys. 81, 3684 (1984). 
[53] V. V. Brazhkin, Y. D. Fomin, A. G. Lyapin, V. N. Ryzhov, and K. Trachenko, Phys. Rev. E 85, 31203 (2012).

[54] K. Trachenko, V. V. Brazhkin, and D. Bolmatov, Phys. Rev. E 89, 032126 (2014).

[55] C. H. Reinsch, Numer. Math. 10, 177 (1967).

[56] S. Jahangiri and H. Behnejad, J. Mol. Liq. 222, 733 (2016).

[57] M. Bethkenhagen, M. French, and R. Redmer, J. Chem. Phys. 138, 234504 (2013).

[58] Y. Song and E. A. Mason, J. Chem. Phys. 91, 7840 (1989).
[59] M. Celli, U. Bafile, G. Cuello, F. Formisano, E. Guarini, R. Magli, M. Neumann, and M. Zoppi, Phys. Rev. B 71, 14205 (2005).

[60] A. H. Narten, E. Johnson, and A. Habenschuss, J. Chem. Phys. 73, 1248 (1980).

[61] L. Temleitner, L. Pusztai, and Y. Akahama, Phys. Rev. B 78, 014205 (2008).

[62] G. Weck, P. Loubeyre, J. H. Eggert, M. Mezouar, and M. Hanfland, Phys. Rev. B 76, 54121 (2007).

[63] A. B. Bhatia and N. H. March, Phys. Chem. Liq. 13, 313 (1984).

[64] C. Cavazzoni, G. L. Chiarotti, S. Scandolo, E. Tosatti, M. Bernasconi, and M. Parrinello, Science 283, 44 (1999). 